\title{
Auditory contralateral induction: An early stage in binaural processing
}

\author{
RICHARD M. WARREN and JAMES A. BASHFORD \\ University of Wisconsin-Milwaukee, Milwaukee, Wisconsin 53201
}

\begin{abstract}
Binaural interaction was studied using headphones presenting signals (tones or filtered speech) to one ear and noises of various spectral compositions to the other. Every half-second, the sides receiving the signal and noise were reversed. The noise was always perceived to alternate from side to side, but the signal appeared to be stationary and diffusely localized about the midsaggital plane when the noise contained the spectral components of the signal at appropriate intensity levels. This delateralization of a monaural signal results from a process called "contralateral induction" (CI). Additional observations indicate that CI corresponds to an early stage in binaural interaction which generally escapes notice because of further perceptual processing.
\end{abstract}

Environmental noises often interfere with signals, and mechanisms minimizing such interference can be of help in auditory perception. The present study, dealing with binaural interaction of signals with noises, was suggested by earlier observations indicating that listeners can effectively cancel some effects of masking.

Previous studies in this laboratory have shown that, under certain conditions, a brief loud sound can evoke illusory detection of a sound it either masks or completely replaces. Thus, when one or more phonemes within a sentence are replaced by a cough or other masking noise (but not a silent gap), the missing portion of speech is clearly "heard," and the listener is unable to detect which portion is absent (Warren, 1970; Warren \& Sherman, 1974). Also, when two intensity levels of the same noise are alternated, the fainter level appears to be on continuously with the rhythmical addition of a pulsed noise (Warren, Obusek, \& Ackroff, 1972). Finally, when a tone is alternated with a louder sound of different spectral composition, the tone may appear to be continuous (Elfner, 1971; Miller \& Licklider, 1950; Thurlow, 1957). Warren et al. (1972) found a close relation between the ability of a louder sound to induce such illusory continuity, or "temporal auditory induction," and the ability of the same sound to mask the fainter sound when both were presented simultaneously. It was concluded that temporal auditory induction of the fainter sound occurred when: (1) there was contextual evidence indicating that a sound might be present along with a louder sound, and (2) the peripheral neural units stimulated by the louder

This study was supported by NIH Grant HD07855 and by the University of Wisconsin-Milwaukee Graduate School. Valuable assistance was provided by Leong $F$. Tai. sound included those which would be stimulated by the anticipated fainter sound. Somewhat similar conclusions have been reached by Houtgast (1972). It should be noted that, while temporal induction is studied in the laboratory as an illusion which produces apparent continuity of an absent sound, in everyday life it can prevent error by permitting veridical perception of continuity of a sound behind a brief masker. There is another type of error which could be produced by noises encountered in everyday life. A noise on one side could cause asymmetrical masking of a signal originating at another spatial position, and without a lateral restoration, or "contralateral induction" (CI), localization to the unmasked side would occur whether appropriate or not. Stated in a somewhat different fashion, if dichotic stimuli are to be used effectively in an acoustically complex environment, it is necessary to isolate components which could correspond to the same source from the inputs to each ear and to combine appropriately these bilateral components.

In everyday life, contralateral induction and subsequent binaural processing usually leads to accurate sound localization, but laboratory studies of $\mathrm{CI}$ should show illusory shifts of monaural sounds away from the stimulated side upon presentation of qualitatively different sounds to the other ear. Reports of such shifts have appeared in the literature from time to time. Using headphones, Egan (1948) found monaural speech to be displaced toward the side of a contralateral noise, and Thurlow and Elfner (1959) found that a monaural tone could be "pulled in" by a contralateral monaural tone of different frequency. Related pulling effects were observed by Butler and Naunton $(1962,1964)$ when stimulating simultaneously with a monaural headphone and a moveable loudspeaker.

If these illusory shifts resulted from a contralateral induction normally leading to more accurate percep- 
tion we would expect a spectral selectivity for $\mathrm{CI}$ resembling that found for temporal induction, since both these types of perceptual restoration are appropriate only if the signal could be incorporated within, or masked by, the other sound. The present study was designed to test whether such spectral criteria do indeed govern $\mathrm{CI}$ and has examined the relation of $\mathrm{CI}$ to binaural interaction in general.

Experiments dealing with lateralized sounds can be complicated by an apparent drift in location over time. Extended listening to a sound heard off to one side is accompanied by an apparent shift toward the midsaggital plane, and other sounds heard subsequently which would normally appear at midplane are displaced away from the side stimulated previously (Bartlett \& Mark, 1922; Flugel, 1920). We reasoned that such drifts could be minimized by periodically switching ears. Such switching had another very useful feature: preliminary testing with monaural tone and contralateral white noise which alternated sides every $500 \mathrm{msec}$ showed that when CI occurred, this signal appeared stationary with a diffuse position centered on the midsaggital plane. The illusion of a stationary signal disappeared abruptly when the signal intensity exceeded the limiting value for CI (noise intensity being fixed), with the signal as well as the noise being perceived to alternate from side to side. These procedural advantages led us to use a periodic reversal of sides (as shown in Figure 1) throughout our study. However, it should be kept in mind that such alternation is an aid to accurate threshold measurement, but is not necessary for CI to occur.

\section{EXPERIMENT 1 CONTRALATERAL INDUCTION OF TONES FROM NOISES}

\section{Subjects and Apparatus}

Nine students from the introductory psychology course were paid to serve as subjects. They were selected from a larger group on the basis of normal Békésy audiograms for both ears and consistency of successive threshold tracings as described in the Procedure section.

A Békésy audiometer (Grason-Stadler Model E-800) was used for generating tonal stimuli for measurements of thresholds, masking, and contralateral induction. The audiometer was set for a sweep rate of 1 octave/min (frequency range from 200 to $8,000 \mathrm{~Hz}$ ) and an intensity change of $2.5 \mathrm{~dB} / \mathrm{sec}$. The subject controlled intensity levels with the standard Békésy switchdepressing the button switch caused the intensity to decrease and releasing the button caused the intensity to increase at the preset rate. Listening was through matched headphones furnished with the audiometer, and levels at the headphones were checked using a Bruel and Kjaer Model 2204 sound. level meter with a 6-cc coupler. Tracings produced by the audiometer provided a record of all responses. Except when obtaining the audiograms needed for screening of subjects, a Grason-Stadler electronic switch Model 829E reversed input to the two ears at $0.5-\mathrm{sec}$ intervals with a rise/decay time of $18 \mathrm{msec}$. Recordings on a fourtrack Ampex 440B provided the three noise stimuli as needed:
(1) white noise; (2) $1 / 3$ octave band noise with slopes of $32 \mathrm{~dB} /$ octave centered on $1,000 \mathrm{~Hz}$; (3) band-reject noise derived from white noise, with an attenuation of $40 \mathrm{~dB}$ for the octave centered logarithmically at $1,000 \mathrm{~Hz}(707$ to $1,414 \mathrm{~Hz})$, with slopes of about $80 \mathrm{~dB} /$ octave at the edges of the rejected octave. The narrow band and band-reject noises were identical with those used in the earlier study on auditory temporal induction (Warrren et al., 1972). All three noises were always presented at an overall sound pressure level of $80 \mathrm{~dB}$ re: $20 \mu \mathrm{N} / \mathrm{m}^{2}$.

\section{Procedure and Results}

As part of the screening procedure, all subjects had threshold tracings determined for each ear, both in order of increasing and of decreasing frequency sweeps. Subjects having a tracing for either ear differing by more than $20 \mathrm{~dB}$ from normal threshold at any frequency, or tracings for any frequency differing more than $15 \mathrm{~dB}$ between successive ascending and descending frequency sweeps, were rejected.

Subjects who met the qualifying criteria returned for three 45-min sessions held on separate days. During these sessions, both ascending and descending frequency sweeps were used with each subject, and the direction of sweeps was balanced in order of presentation as closely as possible for individual subjects and for the group.

\section{Session 1: Monaural Detection and Delateraliza- tion Thresholds in the Presence of Contralateral White Noise}

The subjects always heard monaural white noise at the SPL of $80 \mathrm{~dB}$ while adjusting the intensity of tones swept in frequency by the Békésy audiometer and delivered to the other ear. Switching of the sounds delivered to each ear occurred regularly each $500 \mathrm{msec}$, as shown in Figure 1, for measurement of both detection and contralateral induction (delateralization).

For determination of detection threshold, subjects adjusted the tonal intensity so that it remained just audible in the presence of the contralateral noise. After these judgments were made with both decreasing and increasing frequency sweeps, the limits of contralateral induction were obtained by instructing subjects to adjust the intensity of the tone to the highest level at which it did not seem to move (at higher levels, the tone was perceived to switch from side to side). The switching of the contralateral noise from side to side was detectable

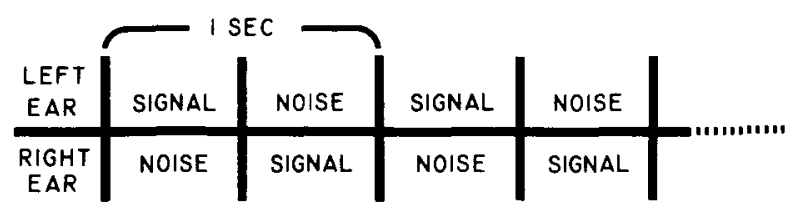

Figure 1. Alternating pattern of dichotic stimulation used with signals consisting of tones (Experiment 1) and filtered voice (Experiment 2). 
at all times. For each tonal frequency, there was a range of supraliminal intensities within which the tone did not seem to alternate in position. The thresholds (in sound pressure level re: $20 \mu \mathrm{N} / \mathrm{m}^{2}$ ) for both detection and contralateral induction are shown in Figure 2. In constructing Figure 2, values were read to the nearest $0.5 \mathrm{~dB}$ from the Békésy audiograms for each of the 24 frequencies represented in the figure. Every data point corresponds to the mean of 18 values (both ascending and descending frequency sweeps for each of the nine subjects). It can be seen that $\mathrm{CI}$ took place at levels well above detection threshold for all tonal frequencies, and hence that the illusory positional stability of the tones was not a consequence of low audibility. Listeners reported that during $C I$ the monaural tone was not localized sharply-its location seemed diffuse and symmetrical relative to the median plane.

\section{Session 2: Monaural Detection and Delateral- ization Thresholds in the Presence of a Contra- lateral Narrow Band Noise}

The procedure was the same as that of Session 1, except that the white noise was replaced by a narrow band noise centered at $1,000 \mathrm{~Hz}$ having the same SPL. Results for both detection and CI are shown in Figure 2. It can be seen that the tonal frequencies with the highest CI levels coincided with the spectral energy peak of the narrow band noise. No corresponding dependence upon the contralateral noise spectrum was observed for the detection threshold. At the center frequency of the noise band $(1,000 \mathrm{~Hz}) \mathrm{CI}$ extended up to a tonal intensity of $49 \mathrm{~dB}$ above the detection threshold, while at higher and lower frequencies CI occurred to a lesser extent.

\section{Session 3: Monaural Detection and Delateraliza- tion Thresholds in the Presence of a Contralateral Band-Reject Noise}

The procedure was the same as that of Sessions 1 and 2 except that the $80-\mathrm{dB}$ SPL noise was derived from white noise with an attenuation of $40 \mathrm{~dB}$ for the octave centered at $1,000 \mathrm{~Hz}$. Results obtained for both detection and CI are shown in Figure 2. It appears that, as in Session 2 using narrowband noise, the shape of the CI curve was determined by the spectral distribution of energy within the noise. The sharp dip in CI observed for the octave centered on $1,000 \mathrm{~Hz}$ corresponded to the lack of energy at these frequencies. A much shallower dip can be seen for detection thresholds at the rejected noise frequencies. At lower and higher frequencies, the spectrum of this band-reject noise resembled that of the white noise used in Session 1 , and it can be seen in Figure 2 that the effect on CI was quite similar for these two noises at frequencies away from the rejected band.

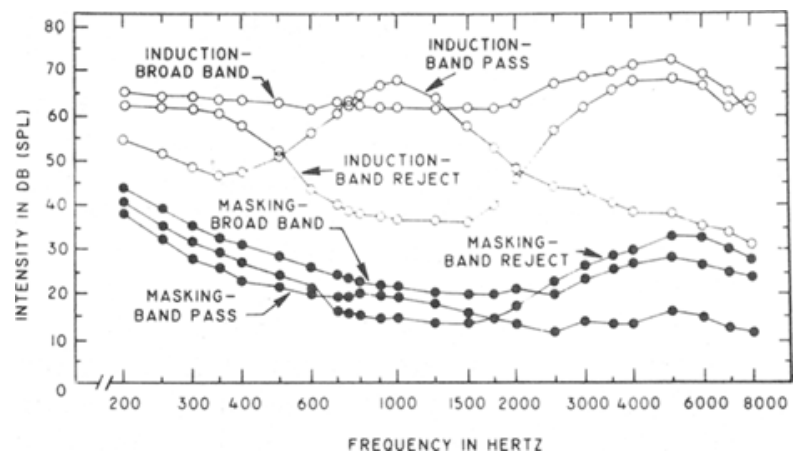

Figure 2. Contralateral induction thresholds and detection thresholds (in sound pressure level) for tones presented with one of three contralateral 80-dB SPL noises: broad-band noise, narrow-band (band pass) noise centered at $1,000 \mathrm{~Hz}$, and band reject noise (rejected frequency band centered at $1,000 \mathrm{~Hz}$ ).

\section{DISCUSSION}

The three lower curves in Figure 2 show detection thresholds for monaural tones from 200 to $8,000 \mathrm{~Hz}$ in the presence of masking by each of the three contralateral noises. Various types of masking were involved: physical masking by cross-ear bone and air conduction, central simultaneous contralateral masking, and ipsilateral backward and forward masking accompanying the switching of sides receiving tone and noise. These masked thresholds were generally between 10 and $20 \mathrm{~dB}$ above ISO normal thresholds (our subjects had been screened for normal thresholds using Békésy audiograms). For the purposes of our experiment, it was not necessary to separate the contributions of the different types of masking upon detection thresholds. However, it should be noted that these masked threshold curves did not reflect the spectral dips and peaks of the contralateral noises to the same extent as the upper curves showing the limits for contralateral induction. Examination of the $\mathrm{CI}$ curves for the different noise conditions suggests that a frequency overlap between tone and noise increases the sensation level at which $\mathrm{CI}$ occurs. For example, at $1 \mathrm{kHz}$, the difference between detection threshold and $\mathrm{CI}$ limit is $22 \mathrm{~dB}$ for band-reject noise, $40 \mathrm{~dB}$ for broad-band noise, and $49 \mathrm{~dB}$ for band-pass noise.

This frequency effect is consistent with the hypothesis which led to the present study; that is, noises best able to mask the signal if it were mixed with the noise would be most effective in generating CI. However, since Experiment 1 was limited to one type of signal (tones changing regularly in frequency and intensity), it appeared desirable to extend this study to other signals. We decided to use speech in Experiment 2, not only because of its perceptual importance, but because of its acoustic heterogeneity with abrupt transitions from one sound to another. 


\section{EXPERIMENT 2 CONTRALATERAL INDUCTION OF FILTERED SPEECH USING NOISE BANDS}

Subjects

\section{Method}

Twenty-six students from the introductory psychology course were paid to serve as subjects. None had participated in Experiment 1 . They were selected from a larger group on the basis of the criteria used in Experiment 1 (normal audiograms, consistency of successive threshold tracings, and consistency of response during training).

\section{Apparatus}

In the preliminary audiometric screening, a Békésy audiometer (Grason-Stadler Model 1701) was used to generate tonal stimuli (250 to 8,000 Hz) and to control their intensities. The audiometer was set for a sweep rate of 2 octaves $/ \mathrm{min}$ and an intensity change rate of $5 \mathrm{~dB} / \mathrm{sec}$. A Crown Model 800 recorder provided the speech stimuli (unfiltered speech and two narrow bands of filtered speech with slopes of $48 \mathrm{~dB}$ per octave-one centered at $1,000 \mathrm{~Hz}$ and one centered at $3,000 \mathrm{~Hz}$ ). All speech was derived from a 58-sec recording of speech (read by a male from a section of an article on the philosophical roots of psychology) which was repeated over and over for $30 \mathrm{~min}$. Eight noise stimuli were recorded on separate tracks of an eight-track Ampex $440 \mathrm{C}$ tape recorder, and consisted of broad-band "speech noise" (resembling the average spectrum of speech and originally generated by the Grason-Stadler Model 1701 audiometer) and 1/3 octave bands of noise with slopes of $32 \mathrm{~dB}$ per octave centered on 333 , $577,1,000,1,732,3,000,5,196$, and $9,000 \mathrm{~Hz}$, respectively. The delivery of noise and voice was controlled by Grason-Stadler Series 1200 programming equipment.

\section{Procedure}

The audiometric screening procedure was carried out as in Experiment 1 to ensure that: (a) only people with normal hearing in both ears were used; and (b) only people with stable and reproducible audiograms were used for subsequent experiments.

Subjects who passed the audiometric screening returned for one additional session. They were first trained with monaural broad-band speech and contralateral speech noise. Sides were reversed each $500 \mathrm{msec}$, using electronic switches with rise/decay settings of $10 \mathrm{msec}$. The noise was fixed at $80 \mathrm{~dB}$ SPL, and the speech level increased automatically at $5 \mathrm{~dB} / \mathrm{sec}$ from $0 \mathrm{~dB}$ to a peak level of $80 \mathrm{~dB}$. The process was repeated, with subjects requested this time to raise their hands when the voice reached an intensity at which it seemed to move from side to side (they were told that content and comprehension were irrelevant). All subjects reported hearing both centered and decentered voice. The motor changing the intensity of the speech then was made inoperative, and subjects adjusted the speech level by turning the audiometer's manual intensity control knob (the dial was hidden from view). The experimenter first adjusted the signal level to $0 \mathrm{~dB}$ SPL, and the subject was instructed to adjust the level until the voice just began to move from side to side. After subjects made a judgment, the variable-intensity voice and the 80- $\mathrm{dB}$ noise were turned off, the voice level recorded, and the voice intensity readjusted to $0 \mathrm{~dB}$. This process was repeated for a total of five decentering threshold judgments. Next, subjects were instructed to adjust the speech intensity to a level at which it was just audible, and five such speech-detection threshold judgments were obtained. Only subjects whose last three judgments covered a range of $12 \mathrm{~dB}$ or less for both decentering and detection, respectively, were allowed to continue in the experiment.

Testing with the two filtered speech bands (centered at 1,000 and $3,000 \mathrm{~Hz}$ ) followed, using a procedure similar to that of the training trials. Subjects were instructed to adjust the speech intensity by turning the audiometer knob until the voice just began to move from side to side. The order of speech-band presentation was counterbalanced across subjects. The five noise bands used with the particular speech band (as shown in Figure 3) were presented in pseudorandom order for a total of three judgments with each noise band (with the restrictions that the first noise had the same center frequency as the voice, and that no noise band was presented twice in succession). After completion of these decentering threshold measurements, detection thresholds were obtained using a procedure which differed only in task instructions. The entire procedure for measuring decentering and detection thresholds with various contralateral noise bands was then carried out for the second band of filtered speech. At the completion of the experiment, each subject had made three speech decentering judgments and three speech detection threshold judgments for each of the five contralateral noise bands presented with each of the two filtered speech bands.

\section{RESULTS AND DISCUSSION}

It should be noted that $\mathrm{CI}$ in Experiment 2 (as well as Experiment 1) did not involve fusion into a single sound image. Instead, two spatially separated sounds were heard: a lateralized noise and a nonlateralized tone or voice have a diffuse and vaguely defined position located symmetrically about the median plane.

In Experiment 2, the median of a subject's three judgments was taken as the score for each condition, and the subject's lower limit for decentering (or upper limit for contralateral induction) was expressed in sensation level (SL) or dB above detection threshold. Each data point in Figure 3 corresponds to the mean of the 26 individual values.

As discussed earlier, if $\mathrm{CI}$ requires that the peripheral neural units stimulated by the noise include those corresponding to stimulation by the contralateral signal, then Experiment 2 should demonstrate the following related effects: (1) the greatest CI should be found for each band of filtered speech when its center frequency matched that of the contralateral noise band, and CI should decrease with increasing frequency separation of the speech and noise

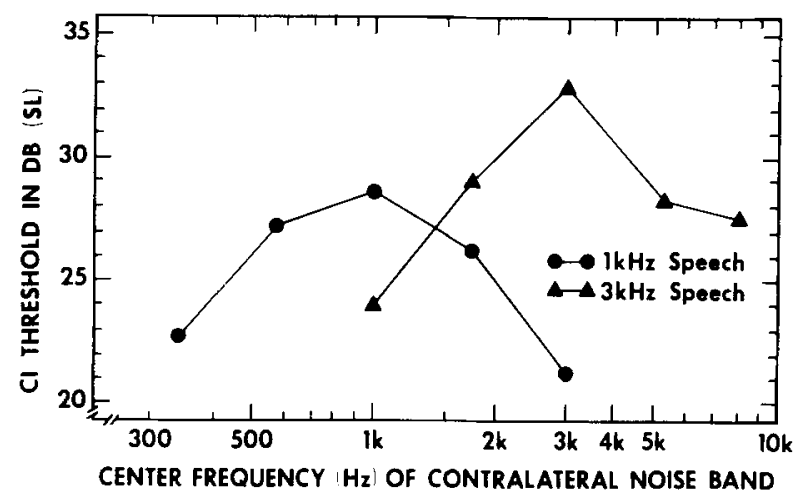

Figure 3. Contralateral induction thresholds (in sensation level) for filtered speech bands centered at 1,000 and $3,000 \mathrm{~Hz}$, each presented with contralateral $80 \mathrm{~dB}$ (sound pressure level) narrow-band noises of various center frequencies. 
bands; (2) the effect of $1,000-\mathrm{Hz}$ narrow band noise should be greater for the filtered speech centered at $1,000 \mathrm{~Hz}$ than at $3,000 \mathrm{~Hz}$, and, correspondingly, the effectiveness of the $3,000-\mathrm{Hz}$ narrow band noise should be greater for the filtered speech at $3,000 \mathrm{~Hz}$ than at $1,000 \mathrm{~Hz}$. Figure 3 shows that these differences were all observed qualitatively, and statistical analyses demonstrated the significance of most of the anticipated effects. A treatment by subjects analysis of variance showed $\mathrm{CI}$ of the speech stimuli to vary as a function of noise-band frequency for both the $1-\mathrm{kHz}(\mathrm{F}=9.70)$ and the $3-\mathrm{kHz}(\mathrm{F}=9.02)$ speech bands ( $\mathrm{df}=4 / 100, p<.001$ for both). Subsequent Tukey HSD tests showed CI to be significantly greater for each of the filtered speech stimuli with the narrow-band noise matched in frequency than with the highest and lowest noise frequencies used with the particular speech band $(\mathrm{df}=100, \mathrm{p}<.01)$. An analysis of individual responses obtained with both the 1,000 - and $3,000-\mathrm{Hz}$ narrow-band noises showed that for each of these noise bands, 20 of the 26 subjects experienced greater $\mathrm{CI}$ effect with the speech band matching the noise band in center frequency than with the other speech band $(p<.005$ by binomial expansion). However, the analysis of variance showed no significant difference between the effects of the $1,000-\mathrm{Hz}$ narrow-band noise on the two speech stimuli, while for the $3,000-\mathrm{Hz}$ narrowband noise the difference between $\mathrm{CI}$ effects was significant $(F=27.24, p<.001)$. This asymmetry in CI effectiveness of narrow-band noise parallels the relative effectiveness of maskers (a signal is masked more efficiently by lower than by higher frequencies; Wegel \& Lane, 1924), and parallels the frequency asymmetry observed for auditory temporal induction (Warren et al., 1972).

The results obtained with filtered voice are consistent with observations made in Experiment 1 for tones. It appears that for both tones and filtered speech, contralateral induction (CI) consists of a cross-ear comparison followed by delateralization of the signal if the contralateral input contains the spectral components of the fainter sound at a sufficiently high intensity.

We made some additional observations with unfiltered voice and broadband noise, using four experienced listeners working in our laboratory. When conditions producing $\mathrm{CI}$ were used and the switching rate was slowed down, delateralization of voice still occurred. Entire sentences could be presented to one side before reversing the speech and noise ears, or switching could even be stopped completely, and the voice still seemed to be located in the median plane. The delateralization threshold remained about the same with slow switching, but was not as sharply defined.

A highly interesting effect was observed when CI conditions were used but switching was made more rapid. When the on-time for voice and noise was decreased from $500 \mathrm{msec}$ to below a threshold value of about $200 \mathrm{msec}$, the single delateralized voice separated into two lateralized voices each saying the same thing and yet not fusing. Additional observations indicated that the splitting of the voice resulted from a form of temporal induction, called "phonemic restoration" (Warren, 1970), produced separately on each side. Starting with conditions producing $\mathrm{CI}$, the input to one ear was shut off and the on-time of the alternating speech and noise heard in the other ear was decreased. When the on-time of each sound fell to about $200 \mathrm{msec}$, listeners heard phonemic restorations on the stimulated side, so that the voice seemed to be on continuously with superimposed noise bursts which did not appear to interrupt the speech. When the switching rate producing monaural phonemic restorations was maintained and dichotic alternation reinstated, independent phonemic restorations occurred on each side and listeners heard the curiously unfused continuous speech described above. These apparently uninterrupted and identical side-by-side voices could be heard down to on-times for speech and noise of about $30 \mathrm{msec}$, beyond which the speech signal became severely distorted by the rapid switching.

It is of interest that the lower temporal limit for CI with voice coincided with the upper temporal limit for phonemic restorations-as if the same noise could not be used for both processes under these conditions.

\section{GENERAL DISCUSSION}

Let us examine the similarities and the differences between contralateral induction $(\mathrm{Cl})$ and temporal induction (TI).

Both CI and TI can act as highly selective mechanisms for extracting masked signals from noise in everyday life. It has been suggested by earlier work that TI leads to perceptual synthesis of an absent signal when peripheral neural units stimulated by a louder sound include those corresponding to an anticipated signal (Warren et al., 1972). The present experiments indicate that an analogous rule governs signal extraction by $\mathrm{CI}$ : If the peripheral neural units stimulated on the side receiving the louder sound include those corresponding to an ongoing contralateral signal, the fainter signal is released from lateralization and moves to a diffuse position located symmetrically about the median plane.

While CI and TI both cancel effects of masking by extracting a possible signal from a potential masker, note that they do so by quite different processes. CI compares two simultaneous inputs, while TI compares the memory trace of a possible signal with current neural activity. The memory 
trace can be simply a steady state sound heard in the immediate past (resulting in illusory continuity of the single sound)-or the memory trace can be quite complex and temporally remote, such as that involved in perceptual restoration of deleted phonemes in accordance with sentence context (Warren, 1970; Warren \& Sherman, 1974).

Experiments producing CI described thus far have used qualitatively different sounds delivered simultaneously to the two ears, since two binaural stimuli which are closely related acoustically would be subject to further processing eventuating in binaural fusion. However, there is an interesting stimulus pairing, rather useful for our purposes, in which signals presented simultaneously to the two ears are matched in long-term spectra, and yet do not fuse. The diffuse localization described in the literature for uncorrelated dichotic white noise indicates that binaural processing is frozen at the stage of $\mathrm{CI}$. The sound seems to be located symmetrically about the median plane, and resembles the effect of standing under a waterfall or hearing rain on a tin roof (David, Guttman, \& van Bergeijk, 1958; Kock, 1950). We decided to use uncorrelated dichotic wide-band noises having very carefully matched spectral composition but different intensities, with the ear receiving the louder noise changing every $500 \mathrm{msec}$. If $\mathrm{CI}$ is a subtractive process with delateralization requiring removal of components corresponding to the fainter side from the louder side, listeners should hear complete delateralization only for the fainter noise, with a residue corresponding to the intensity difference lateralized at the side receiving the louder noise. When five listeners (graduate students and laboratory staff) heard a noise at $80 \mathrm{~dB}$ SPL on one side and an uncorrelated spectrally matched noise differing by $1 \mathrm{~dB}$ or more on the other side, in addition to the dichotic undera-waterfall effect, a faint monaural residue was heard to alternate from side to side in synchrony with the noise of higher intensity. The separation into a diffuse nonlateralized sound and a sharply lateralized residue was perceived clearly by all. Presumably, a similar subtraction of components from contralateral noise took place with the tone and voice signals we used earlier, but the effects of removing components were much more obvious when noises of equivalent spectral composition and nearly equal intensities appeared on both sides.

Contralateral induction seems to reflect general processes underlying binaural localization. It appears that CI corresponds to an early stage in localization, involving information sufficient to indicate the possibility of bilateral acoustic input from a single source, but insufficient to bring about fusion. Further processing of binaural input resulting in a sharply localized image involves such factors as: (1) appropriate relative intensities at the two ears for each of the spectral components corresponding to a single source; (2) appropriate phase or time-ofarrival relations at the two ears; and (3) changes in (1) and (2) correlated with head movements (for reviews of cues involved in binaural fusion and lateralization, see Henning, 1975; Mills, 1972).

In all of our experiments with CI, we have delivered only one sound at a time to each ear, and so have been dealing with the simplest arrangement for binaural interaction. If more than one sound is introduced to at least one ear, we create more complex conditions which can lead to masking level differences (MLDs), the subject of a vast literature over the last few decades (see Studebaker, 1973). MLDs correspond to a decrease in masked threshold when two ears are used rather than one, and permit a signal to be detected binaurally when it could not be detected in the input to either ear heard alone. As noted above, MLDs require more complex stimuli than we have used in our experiments with $\mathrm{CI}$, and involve an auditory processing beyond that required for $\mathrm{CI}$. However, the subtractive interactions of $C I$ could be involved as the first stage in the unmasking of an otherwise undetectable signal, and indeed theories considering that similar subtractive interactions are involved in MLDs have been proposed (see Durlach, 1963, 1972; Jeffress, 1972).

We suggest that contralateral induction is a general early stage, which compares input to the two ears and segregates those contralateral components which could correspond to a single source. Contralateral induction has characteristics in common with temporal induction, which compares and segregates components separated in time. While both CI and TI can be studied conveniently as laboratory illusions, outside the laboratory they usually do not lead to error, but rather aid in avoiding errors which would otherwise accompany the masking produced in complex acoustic environments.

\section{REFERENCES}

Bartlett, F. C., \& MARK, H. A. A note on local fatigue in the auditory system. British Journal of Psychology, 1922, 13, 215-218.

Butler, R. A., \& Naunton, R. F. Some effects of unilateral auditory masking upon the localization of sound in space. Journal of the Acoustical Society of America, 1962, 34, 1100-1107.

Butter, R. A., \& Naunton, R. F. Role of stimulus frequency and duration in the phenomenon of localization shifts. Joumal of the Acoustical Society of America, 1964, 36, 917-922.

David, E. E., Guttman, N., \& van Bergeijk, W. A. On the mechanism of binaural fusion. Journal of the Acoustical Society of America, 1958, 30, 801-802.

DURLACH, N. I. Equalization and cancellation theory of binaural masking-level differences. Journal of the Acoustical Society of America, 1963, 35, 1206-1218.

DurLACH, N. I. Binaural signal detection: Equalization and cancellation theory. In J. V. Tobias (Ed.), Foundations of modern auditory theory (Vol. 2). New York: Academic Press, 1972.

EGAN, J. P. The effect of noise in one ear upon the loudness of 
speech in the other. Journal of the Acoustical Society of America, 1948. 20, 58-62.

ELFNER, L. F. Continuity in alternately sounded tonal signals in a free field. Journal of the A coustical Society of America, 1971, 49, 447-449.

Flugel, J. C. On local fatigue in the auditory system. British Journal of Psychology, 1920, 11, 105-134.

Henning, G. B. Auditory localization. In M. S. Gazzaniga \& C. Blakemore (Eds.), Handbook of psychobiology. New York: Academic Press, 1975.

Houtgast, T. Psychophysical evidence for lateral inhibition in hearing. Journal of the Acoustical Society of America, 1972, 51, 1885-1894.

JefrRess, L. A. Binaural signal detection: Vector theory. In J. V. Tobias (Ed.), Foundations of modern auditory theory (Vol. 2). New York: Academic Press, 1972.

KocK, W. E. Binaural localization and masking. Joumal of the Acoustical Society of America, 1950, 22, 801-804.

Miller, G. A., \& LICKLIDER, J. C. R. The intelligibility of interrupted speech. Joumal of the Acoustical Society of America, 1950, 22, 167-173.

Mrlls, W. A. Auditory localization. In J. V. Tobias (Eds.), Foundations of modem auditory theory (Vol. 2). New York: Academic Press, 1972.
Studebaker, G. A. Auditory masking. In J. Jerger (Ed.), Modern developments in audiology (2nd ed.). New York: Academic Press, 1973.

Thurlow, W. R. An auditory figure-ground effect. American Journal of Psychology, 1957, 70, 653-654.

Thurlow, W. R., \& ElFner, L. F. Continuity effects with alternately sounding tones. Journal of the Acoustical Society of America, 1959, 31, 1337-1339.

W ARREN, R. M. Perceptual restoration of missing speech sounds. Science, 1970, 167, 392-393.

WarRen, R. M., Obusek, C. J., \& ACkroff, J. M. Auditory induction: Perceptual synthesis of absent sounds. Science, 1972, 176, 1149-1151.

Warren, R. M., \& Sherman, G. L. Phonemic restorations based on subsequent context. Perception \& Psychophysics, 1974, 16, $150-156$.

WEGEL, R. L., \& LANE, C. E. The auditory masking of one pure tone by another and its probable relation to the dynamics of the inner ear. Physical Review, 1924, 23, 267-285.

(Received for publication March 19, 1976; revision accepted September $14,1976$. 